\title{
The spectral decomposition of Active Galactic Nuclei with double-peaked Balmer lines from SDSS
}

\author{
W. Bian \\ Department of Physics and Institute of Theoretical Physics, Nanjing Normal University, \\ Nanjing 210097, China
}

\begin{abstract}
For a sample of 52 double-peaked broad-lines AGNs from the Sloan Digital Sky Survey (SDSS), we do the spectral decomposition to obtain the host spectrum and the nuclei spectrum from their SDSS spectra, as well as the bulge luminosity $\left(L_{b u l g e}\right)$, stellar velocity dispersion $\left(\sigma_{*}\right)$. A strong correlation is found between the $\sigma_{*}$ and the gaseous velocity dispersion in narrow line regions. With the $M_{\mathrm{bh}}-\sigma_{*}$ relation, we estimate the black hole masses, range from $1.0 \times 10^{7}$ to $6 \times 10^{8} \mathrm{M}_{\odot}$, and the Eddington ratio from about 0.01 to about 1 . It is consistent with the result from $M_{\mathrm{bh}}-L_{\mathrm{bulge}}$ relation. However, it is not consistent with the mass from the $\mathrm{H} \beta$ FWHM. It seems that the empirical size-luminosity relation for broad line regions dose not hold for double-peaked AGNs, otherwise the calibration factor is as small as 0.185, suggesting the non-virial dynamics of broad line regions.
\end{abstract}

Keywords. galaxies:bulges — galaxies:active — galaxies: nuclei — black hole physics

\section{The model of stellar contribution in double-peaked AGNs}

With the Sloan Digital Sky Survey (SDSS), more double-peaked broad Balmer emission profiles have been detected, about 3\% of the $z<0.332$ SDSS AGNs (e.g. Eracleous \& Halpern 2003; Strateva et al. 2003). It is found that about $50 \%$ starlight contribution in optical continuum around $\mathrm{H} \alpha$. It remains a matter as debate of the origin of the double-peaked profiles.

Here we used the sample of double-peaked AGNs at $z<0.332$ from SDSS (Strateva et al. 2003) to investigate their host properties. Through the stellar population synthesis code, STARLIGHT (version 2.0, Cid Fernandes et al. 2001), we modelled the stellar contribution in the SDSS double-peaked spectra. We obtain 52 double-peaked AGNs with reliable $\sigma_{*}$ measurements of stellar velocity dispersions considering the resolution of SDSS spectra and the template spectra. For more detail, please refer to Bian et al. (2007).

The typical uncertainty of $\sigma_{*}$ is based upon the effective $\mathrm{S} / \mathrm{N}$ at $4020 \AA$. In our sample, the effective mean spectral $\mathrm{S} / \mathrm{N}$ at $4020 \AA$ for these objects are 7.2. The typical uncertainty in $\sigma_{*}$ should be around $20 \mathrm{~km} \mathrm{~s}^{-1}$.

$\sigma_{*}$ is measured for 48 objects with SDSS spectra not covering Ca II $\lambda \lambda$ 8498, 8542, 8662 triplet. We also use the synthesis method to the sample of Greene \& Ho (2006) in two manners: one is using whole spectrum, the other is just using partial spectrum between $3200 \AA$ and $7500 \AA$ at the rest frame. And the the values of $\sigma_{*}$ in these two manners are similar. We obtain the correction formula and do the correction of $\sigma_{*}$ measured from the spectra not including Ca II $\lambda \lambda$ 8498, 8542, 8662 triplet for 48 double-peaked AGNs.

For these 52 double-peaked SDSS AGNs, the host luminosity in V band is calculated from optical monochromatic luminosity and the stellar fraction at $5530 \AA$. We assume 
that the host luminosity in $\mathrm{V}$ band approximates the bulge luminosity $L_{\text {bulge }}$ in $\mathrm{V}$ band. We then use the following formula to calculate the bulge mass: $\log \left(M_{\text {bulge }} / \mathrm{M}_{\odot}\right)=$ $1.18 \log \left(L_{\text {bulge }} / \mathrm{L}_{\odot}\right)-1.11$ (Magorrian et al. 1998). For the relation between this bulge mass and the black hole mass from $\sigma_{*}^{c}$, we fit with fixed slope as 1 , intercept is $2.93 \pm 0.05$, correlation coefficient is 0.53 . The null hypothesis is less then $10^{-4}$. It is suggested that the black hole mass is about 0.002 of the bulge mass. Therefore, the SMBH mass from $L_{\text {bulge }}$ and the Magorrian relation agrees with that from $\sigma_{*}^{c}$. The image decomposition of double-peaked AGNs will be discussed in the future.

\section{The relation between the stellar and gaseous velocity dispersions}

The gaseous velocity dispersion $\left(\sigma_{\mathrm{g}}\right)$ is obtained from our fitting of the core [O III $] \lambda 5007$ line after correction of the SDSS spectral resolution. For the relation between $\sigma_{\mathrm{g}}$ and $\sigma_{*}^{c}$ for these 52 double-peaked AGNs, the best linear fit gives: $\sigma_{\mathrm{g}}=(-29.9 \pm 28.6)+(1.17 \pm$ $0.17) \sigma_{*}^{\mathrm{c}}$, the correlation coefficient is 0.7 . We can use the FWHM of the core [O III] line to trace the stellar velocity dispersion. This strong correlation is consistent with those of other type AGNs (e.g. nearby Seyfert galaxies, Type II AGNs), implying that the gaseous kinematics of narrow line regions in double-peaked AGNs are similar to other type AGNs, primarily dominated by the bulge gravitational potential (e.g. Nelson \& Whittle 1996).

\section{The SMBHs masses and Eddington ratios in double-peaked AGNs}

With the relation, $M_{\mathrm{bh}}\left(\sigma_{*}^{c}\right)=10^{8.13}\left(\sigma_{*}^{c} / 200 \mathrm{~km} \mathrm{~s}^{-1}\right)^{4.02} \mathrm{M}_{\odot}$ (e.g. Tremaine et al. 2002 and reference therein), we calculate the SMBH mass and then the Eddington ratio. We use the monochromatic luminosity at $5100 \AA$ to estimate the bolometric luminosity, $L_{\mathrm{bol}}=9 \times \lambda L_{\lambda}(5100 \AA)$.

For the typical uncertainty of $20 \mathrm{~km} \mathrm{~s}^{-1}$ for $\sigma_{*}=200 \mathrm{~km} \mathrm{~s}^{-1}$, the error of $\log \sigma_{*}$ would be about 0.05 dex, corresponding to 0.2 dex for $\log M_{\mathrm{bh}}$. The error of $\log M_{\mathrm{bh}}$ is about 0.4 considering the error of 0.3 dex form the $M_{\mathrm{bh}}-\sigma_{*}$ relation (Tremaine et al. 2002). Richards et al. (2006) suggested a bolometric correction factor of $10.3 \pm 2.1$ at $5100 \AA$. Therefore, the final Eddington ratio, $L_{\mathrm{bol}} / L_{\mathrm{Edd}}$, has a large uncertainty, about 0.5 dex.

The black hole masses $\log M_{\mathrm{bh}} / \mathrm{M}_{\odot}$ are estimated from $\sigma_{*}^{c}$, ranging from 7 to 8.7 , with a mean value of $7.76 \pm 0.37$. The Eddington ratio is from 0.01 to 1 and the mean value is -1.13 . It was suggested that the accretion disk in double-peaked AGNs is in the mode of Advection Dominated Accretion Flow (ADAF) (e.g. Eracleous \& Halpern 2003). The higher Eddington ratios clearly show that these double peaked AGNs have accretion disks in the standard regime.

This work has been supported by the NSFC (No. 10473005) and the Science-Technology Key Foundation from Education Department of China (No. 206053).

\section{References}

Bian W., Chen Y., Qu Q., \& Wang J. 2007, ApJ, in press, astro-ph/0706.2473

Cid Fernandes R., Sodre L., Schmitt H. R., \& Leao J. R. S. 2001, MNRAS 325, 60

Eracleous M. \& Halpern J. P. 2003, ApJ, 599, 886

Greene J. E. \& Ho L. C., 2006, ApJ, 641, L21

Magorrian J., et al. 1998, AJ, 115, 2285

Nelson C. H. \& Whittle M., 1996, ApJ, 465, 96

Richards G. T., et al. 2006, ApJS, 166, 470

Strateva I. V., et al. 2003, AJ, 126, 1720

Tremaine S., et al. 2002, ApJ, 574, 740 